\title{
Reflexos da Ansiedade e Depressão na Qualidade de Vida de Pacientes Estomizados
}

\author{
Mariana Fröhlich Alievi, ${ }^{1}$ Marli Maria Loro, ${ }^{2}$ Ligia Beatriz Bento Franz, ${ }^{2}$ \\ Pâmella Pluta, ${ }^{2}$ Adriane Cristina Bernat Kolankiewicz ${ }^{3}$
}

\begin{abstract}
RESUMO
Objetivo: Avaliar a autoeficácia, qualidade de vida, ansiedade e depressão em estomizados com câncer colorretal. Método: Estudo transversal realizado com estomizados de um município do Estado do Rio Grande do Sul, Brasil. A coleta de dados foi por meio de questionário contendo condições sociodemográficas e escalas de qualidade de vida, autoeficácia geral percebida e hospitalar de ansiedade e depressão. Resultados: A autoeficácia foi satisfatória. O domínio mais elevado da qualidade de vida foi o das relações sociais e o menor, o domínio físico, mas em todos os domínios ela se mostrou satisfatória. Cerca de $24 \%$ apresentaram ansiedade e $20 \%$ depressão. Houve correlação significativa positiva entre autoeficácia e qualidade de vida. Ansiedade, depressão e qualidade de vida apresentam correlação negativa. Pacientes com elevados níveis de ansiedade e depressão apresentam baixa qualidade de vida. A correlação de autoeficácia e ansiedade e depressão não foi significativa, existindo uma relação de independência entre as variáveis. Conclusão: Os estomizados apresentaram boa autoeficácia; qualidade de vida com média mais elevada no domínio das relações sociais e médias intermediárias nos domínios psicológico, físico e meio ambiente. Quanto à ansiedade e depressão, os escores foram baixos, portanto satisfatórios.
\end{abstract}

Palavras-chave: Estoma. Ansiedade. Depressão. Serviços de saúde.

\section{REFLEXES OF ANXIETY AND DEPRESSION INQUALITY OF LIFE OF OSTOMIZED PATIENTS}

\section{ABSTRACT}

Objective: To evaluate the self-efficacy, quality of life, anxiety and depression in patients with colorectal cancer. Method: A cross-sectional study performed with stomates of a city in the State of Rio Grande do Sul / Brazil. The data collection was through a questionnaire containing socio-demographic conditions and quality of life scales, perceived general self-efficacy and hospital anxiety and depression. Results: Auto efficacy was satisfactory. The highest domain of quality of life was social relations and the smallest in the physical domain, but in all domains it was satisfactory. About $24 \%$ had anxiety and $20 \%$ depression. There was a significant positive correlation between self efficacy and quality of life. Anxiety, depression and quality of life have a negative correlation. Patients with high levels of anxiety and depression have low quality of life. The correlation of self efficacy and anxiety and depression were not significant, there is a relationship of independence between variables. Conclusion: The stomates presented good self efficacy; quality of life with higher average in the domain of social relations and intermediate means in the psychological, physical and environmental domains. As for anxiety and depression the scores were low, therefore satisfactory.

Keywords: Stoma. Anxiety. Depression. Health services.

\footnotetext{
Hospital de Caridade de ljuí. ljuí/RS, Brasil.

Universidade Regional do Noroeste do Estado do Rio Grande do Sul (Unijuí). Ijuí/RS, Brasil.

${ }^{3}$ Autora correspondente. Universidade Regional do Noroeste do Estado do Rio Grande do Sul (Unijuí). Rua do Comércio, 3000 - Bairro Universitário - ljuí/RS, Brasil. http://lattes.cnpq.br/7833969359741646. adriane.bernat@unijui.edu.br
} 


\section{INTRODUÇÃO}

$\mathrm{Na}$ contemporaneidade, as mudanças sociodemográficas, econômicas e epidemiológicas ocasionadas pela urbanização acelerada, novos padrões de consumo, avanços científicos e tecnológicos promoveram o aumento da expectativa de vida populacional e longevidade, associados ao aumento de Doenças Crônicas Não Transmissíveis (DCNTs). Isso resulta no aumento dos estomizados no Brasil, e a cada ano realiza-se, aproximadamente, 1 milhão e 400 mil procedimentos cirúrgicos para confecção de estomias (MIRANDA et al., 2016). O Câncer Colorretal (CCR) é uma das principais causas para realização de uma estomia (LUZ et al., 2014).

Ele é considerado a quarta causa de câncer no mundo e no Brasil, bem como de óbitos, e metade dos pacientes morre em menos de cinco anos (ATTOLINI; GALLON, 2010). A sobrevida média global para o CCR é em torno de $59 \%$ em países desenvolvidos e $42 \%$ em países em desenvolvimento, e pode chegar a 90\% se diagnosticada em estágio inicial (BRASIL, 2018).

Os pacientes estomizados por CCR têm sua vida modificada com alterações físicas, emocionais e sociais, fazendo-se necessário aceitar as restrições impostas pela doença para um convívio social saudável (RIBEIRO et al., 2016). A realização da cirurgia para confecção da estomia é um momento conflituoso para o paciente e seus familiares. Ademais, suas relações sociais, assim como as alterações corporais pós-cirurgia perpassam por aspectos fisiológicos, psicológicos, sociais e espirituais que requerem do indivíduo adaptação diante desse novo modo de ser (MONTEIRO et al., 2016).

Isso decorre do fato de a estomia poder ocasionar alterações físicas significativas no corpo, perda da integridade e autonomia, que envolvem dificuldades pessoais e sociais. A convivência com a bolsa de estomia favorece sentimentos conflituosos, preocupações e dificuldades para lidar com a nova situação (MONTEIRO et al., 2016), bem como emergem momentos de ansiedade e depressão (PATRÃO; ALVES NEIVA, 2017). Logo, a Qualidade de Vida (QV) está associada ao estado de saúde do estomizado e sua capacidade de viver plenamente (PLETSCH, 2013). Para tanto, além do impacto da estomia, a reabilitação e os aspectos psicossociais também influenciam na QV, sendo necessário o apoio dos profissionais de saúde (GOMBOSKI, 2010). Destaca-se que ainda há uma escassez de estudos que avaliem a QV de estomizados no Brasil (GOMBOSKI, 2010).
A partir desse contexto, e da importância de investigar os aspectos que possam facilitar a adaptação do paciente à estomia, bem como planejar as intervenções que podem ser desenvolvidas nos serviços de saúde, este estudo objetiva avaliar a autoeficácia, qualidade de vida, ansiedade e depressão de pacientes estomizados.

\section{MATERIAL E MÉTODOS}

Trata-se de estudo transversal, com abordagem quantitativa. Realizado com pacientes estomizados cadastrados na Secretaria Municipal de Saúde (SMS) de um município localizado na Região Noroeste do Estado do Rio Grande do Sul (RS), Brasil. A população foi selecionada por meio de uma lista de pacientes cadastrados no Gerenciamento de Usuários com Deficiência (GUD), com a Classificação Internacional de Doenças (CID) de CCR.

Foram incluídos estomizados com diagnóstico médico de CCR com a realização da estomia havia pelo menos 30 dias e excluídos aqueles que não tinham condições psíquicas de responder o questionário, avaliados pela pesquisadora por meio da avaliação da condição psíquica, sendo observadas consciência, atenção, orientação, memória, afetividade, conduta e linguagem.

Inicialmente obteve-se uma lista com 39 pacientes estomizados, com nome completo, endereço e telefone. De acordo com os critérios, 10 (25,6\%), foram excluídos, totalizando uma população elegível de 29 pacientes. Destes, 25 (86\%) participaram do estudo. Para verificar a representatividade estatística do número de pacientes em estudo, adotaram-se como critérios: proporção estimada de $50 \%$, erro amostral de $10 \%$ e nível de significância de $5 \%$. A partir do cálculo do tamanho da amostra seriam necessários, no mínimo, 23 pacientes.

A coleta de dados foi realizada pela pesquisadora e por bolsistas previamente capacitadas para o uso das escalas e padronização das entrevistas. Os pacientes foram contatados por telefone e, no momento, fez-se o convite para participar, bem como o agendamento das entrevistas. Elas ocorreram no domicílio do paciente, em horário e turno de preferência do entrevistado, em uma sala reservada, para garantir a privacidade dos sujeitos. Todos os participantes assinaram o Termo de Consentimento Livre e Esclarecido em duas vias.

A coleta foi realizada por meio de questionários, contendo dados que permitem a caracterização de condições sociodemográficas: sexo, idade, raça au- 
torreferida, estado civil, escolaridade, renda familiar e quantidade de residentes na casa (variáveis independentes), utilizada a escala de QV WHOQOL-bref, autoeficácia geral percebida (EAGP) e escala Hospitalar de Ansiedade e Depressão (HAD).

O WHOQOL-bref foi validado para ser utilizado na população brasileira e apresenta boa consistência interna (FLECK et al., 2000). Conta com 26 questões, duas a respeito da $Q V$ em geral e as demais representam cada uma das 24 facetas que compõem o instrumento original. Estão divididas em quatro domínios: "físico" (dor física e desconforto, dependência de medicação/tratamento, energia e fadiga, mobilidade, sono e repouso, atividades da vida cotidiana, capacidade para o trabalho), "psicológico" (sentimentos positivos e negativos, espiritualidade/crenças pessoais, aprendizado/memória/concentração, aceitação da imagem corporal e aparência, autoestima), "relações sociais" (relações pessoais, atividade sexual, suporte/ apoio social) e "ambiente" (segurança física, ambiente físico, recursos financeiros, novas informações/habilidades, recreação e lazer, ambiente no lar, cuidados de saúde, transporte) (ALMEIDA-BRASIL et al., 2017).

As questões são formuladas para respostas em escalas tipo Likert, incluindo intensidade ("nada" a "extremamente"), capacidade ("nada" a "completamente"), frequência ("nunca" a "sempre") e avaliação ("muito insatisfeito" a "muito satisfeito"; "muito ruim" a "muito bom"). As pontuações de cada domínio foram transformadas numa escala de 0 a 100 e expressas em termos de médias, conforme preconiza o manual produzido pela equipe do WHOQOL, em que médias mais altas sugerem melhor percepção de QV (ALMEIDA-BRASIL et al., 2017).

O questionário de AEGP foi desenvolvido em 1979 no idioma alemão, contendo 20 itens. Em 1981, a escala foi reduzida para 10 itens e, posteriormente traduzida e adaptada para 30 idiomas. 0 instrumento apresenta sólidas evidências de validade e confiabilidade advindas de pesquisas com populações de diferentes países (PATRÃO; ALVES NEIVA, 2017). Sua validação para o uso na população brasileira apresentou características psicométricas adequadas (SOUZA; SOUZA, 2004).

Trata-se de uma escala de autorrelato com 10 itens respondidos em formato Likert, com foco nas crenças e expectativas de autoeficácia relacionadas com a gestão dos sintomas associados à condição de saúde, a procura de estratégias e a confiança nas capacidades para lidar com as dificuldades ou barreiras (PATRÃO; ALVES NEIVA, 2017).
A EAGP é constituída por 10 itens respondidos numa escala com 4 alternativas de resposta ( $1-$ De modo nenhum é verdade; 2 - Dificilmente é verdade; 3 - Moderadamente verdade e 4 - Exatamente verdade), podendo-se obter uma pontuação total entre os 10 e os 40 pontos ou uma pontuação média entre 1 e 4 pontos. Todos os itens encontram-se formulados no sentido positivo, e valores elevados na escala EAGP indicam a presença de uma elevada autoeficácia geral. Trata-se de uma escala unidimensional, na qual os 10 itens convergem na avaliação do constructo global de autoeficácia (SBICIGO et al., 2012).

A escala HAD possui 14 itens, dos quais 7 são voltados para a avaliação da ansiedade (HAD-A) e 7 para a depressão (HAD-D), validada no Brasil por Botega et al., 1995. Cada um dos seus itens pode ser pontuado de zero a 3 , compondo uma pontuação máxima de 21 pontos para cada escala. Para a avaliação da frequência da ansiedade e da depressão foram obtidas as respostas aos itens da HAD. Adotou-se os seguintes pontos de corte HAD-ansiedade: sem ansiedade de 0 a 8; com ansiedade $\geq 9$; HAD-depressão: sem depressão de 0 a 8 ; com depressão $\geq 9$ (MARCOLINO et al., 2007).

Os dados foram organizados no programa Epi-Info ${ }^{\circledast}$ 6.04, com dupla digitação independente. Após correções de erros e inconsistências na digitação, a análise estatística foi realizada no Statistical Package for the Social Sciences (SPSS ${ }^{\circledR}$ ), versão 21.0 for Windows. Utilizou-se a estatística descritiva representada pelas medidas de tendência central (média ou mediana) e de dispersão (desvio padrão e intervalo de confiança de 95\% - IC95\%). As variáveis categóricas foram descritas pelas frequências bruta $(n)$ e relativa (\%). Para a comparação de médias para amostras não paramétricas, utilizou-se o teste de Mann-Whitney ou Kruskal Whallis. A confiabilidade dos instrumentos foi analisada pelo Alfa de Cronbach.

O estudo foi aprovado pelo Comitê de Ética em Pesquisa (CEP) da Universidade de Cruz Alta (Unicruz) sob o Parecer 80479417.2.0000.5322.

\section{RESULTADOS}

Participaram 25 pacientes, com faixas de idade prevalentes de 61 a 70 anos, 36,0\% (n=9); de 51 a 60 anos e de 71 a 80 anos, cada uma representando $20,0 \%(n=5)$ da amostra. A idade média foi 66,9 ( $d p$ $=11,5$ ) anos. 0 número de pacientes do sexo masculino foi $52,0 \%(n=13)$, mostrando-se ligeiramente superior ao sexo feminino, $48,0 \%(n=12)$. O maior percentual dos investigados declarou ser da raça 
branca, 80,0\% ( $n=20)$. Em relação ao estado civil, $56,0 \%(n=14)$ não conviviam em união, eram viúvos/ solteiros/separados.

A ausência de atividade laboral foi confirmada por $80,0 \%(n=20)$. E, quanto ao nível de instrução, $56,0 \%(n=14)$ cursaram até o Ensino Fundamental incompleto, seguido dos níveis de Ensino Médio completo e Superior completo, cada um representando $16,0 \%(n=4)$. Quando questionados sobre o número de pessoas que coabitavam no domicílio, $68,0 \%$ $(n=17)$ informaram residir com três ou mais pessoas. $E$, em relação à renda mensal familiar, $54,2 \%(n=13)$ declararam renda de até $R \$ 2.000,00$. No que se refere ao tempo de estomia, prevaleceu o período de até cinco anos, com 76,0\% ( $n=19)$.

Ao avaliar os resultados da EAGP e o somatório das pontuações, obteve-se o valor médio, que foi de $32,9(d p=7,2)$. Considerando-se que o instrumento é unidimensional, foi estimada a confiabilidade em que os resultados foram apontados como satisfatórios ( $\alpha \mathrm{C}$ $=0,874$ ). (Tabela 1).

Ao avaliar a QV, a média mais elevada foi no domínio das Relações Sociais $(72,7 \pm 10,6)$, enquanto que a menor média foi detectada no domínio Físico $(63,7 \pm 23,8)$. Em relação à confiabilidade, esta mostrou-se satisfatória $(\alpha C \geq 0,700)$ em todos os domínios, sendo a mais elevada no domínio Físico $(\alpha C=$ 0,878 ) enquanto que a menor, no domínio das Relações Sociais $(\alpha C=0,636)$. (Tabela 2$)$.

A Tabela 3 evidencia resultados referentes à ansiedade, verificaram-se o valor médio de $5,5(d p=4,5)$ e mediana de 4,0 pontos. De acordo com o ponto de corte da escala, $24 \%(n=6)$ apresentaram ansiedade. Quanto à depressão, a média foi estimada em 4,9 ( $d p$ $=3,9$ ) com mediana de 3,0 pontos. Os casos com depressão representaram $20 \%(n=5)$ da amostra.

Tabela 1 - Medidas de tendência central e de variabilidade da EAGP, na perspectiva de estomizados. RS, Brasil. 2018

\begin{tabular}{|c|c|c|c|c|c|c|c|}
\hline \multirow[b]{2}{*}{ Escala } & \multirow[b]{2}{*}{ Média } & \multirow{2}{*}{$\begin{array}{l}\text { Desvio } \\
\text { padrão }\end{array}$} & \multicolumn{2}{|c|}{ Amplitude } & \multicolumn{3}{|c|}{ Quartis } \\
\hline & & & Mínimo & Máximo & 10 & $\begin{array}{c}\text { 20 } \\
\text { Mediana }\end{array}$ & 39 \\
\hline Total & 32,9 & 7,2 & 13,0 & 40,0 & 29,5 & 36,0 & 38,0 \\
\hline Média & 3,3 & 0,7 & 1,3 & 4,0 & 3,0 & 3,6 & 3,8 \\
\hline $\begin{array}{l}\text { Mesmo que alguém se oponha eu encontro maneira } \\
\text { e formas de alcançar o que quero }\end{array}$ & 3,2 & 1,1 & 1,0 & 4,0 & 3,0 & 4,0 & 4,0 \\
\hline $\begin{array}{l}\text { Tenho facilidade para persistir em minhas intenções e } \\
\text { alcançar meus objetivos }\end{array}$ & 3,3 & 1,0 & 1,0 & 4,0 & 3,0 & 4,0 & 4,0 \\
\hline $\begin{array}{l}\text { Tenho confiança para me sair bem em situações } \\
\text { inesperadas }\end{array}$ & 3,2 & 1,2 & 1,0 & 4,0 & 3,0 & 4,0 & 4,0 \\
\hline $\begin{array}{l}\text { Devido a minhas capacidades sei como lidar com } \\
\text { situações imprevistas }\end{array}$ & 3,2 & 1,1 & 1,0 & 4,0 & 2,5 & 4,0 & 4,0 \\
\hline $\begin{array}{l}\text { Consigo sempre resolver os problemas difíceis } \\
\text { quando me esforço bastante }\end{array}$ & 3,3 & 1,0 & 1,0 & 4,0 & 3,0 & 4,0 & 4,0 \\
\hline $\begin{array}{l}\text { Eu me mantenho calmo mesmo enfrentando } \\
\text { dificuldades porque confio na minha capacidade de } \\
\text { resolver problemas }\end{array}$ & 3,2 & 1,2 & 1,0 & 4,0 & 2,5 & 4,0 & 4,0 \\
\hline $\begin{array}{l}\text { Quando eu enfrento um problema geralmente } \\
\text { consigo encontrar diversas soluções }\end{array}$ & 3,3 & 1,1 & 1,0 & 4,0 & 3,0 & 4,0 & 4,0 \\
\hline $\begin{array}{l}\text { Se estou com problemas geralmente encontro uma } \\
\text { saída }\end{array}$ & 3,4 & 0,9 & 1,0 & 4,0 & 3,0 & 4,0 & 4,0 \\
\hline $\begin{array}{l}\text { Não importa a diversidade eu geralmente consigo } \\
\text { enfrentá-la }\end{array}$ & 3,4 & 0,9 & 1,0 & 4,0 & 3,0 & 4,0 & 4,0 \\
\hline
\end{tabular}


Tabela 2 - Distribuição dos escores médios da QV geral e por domínio do WHOQOL-bref na perspectiva de estomizados. RS, Brasil. 2018

\begin{tabular}{|c|c|c|c|c|c|c|c|c|}
\hline \multirow[b]{2}{*}{ Qualidade de vida geral e domínios } & \multirow[b]{2}{*}{ Média } & \multirow[b]{2}{*}{ DP } & \multicolumn{2}{|c|}{ Amplitude } & \multicolumn{3}{|c|}{ Quartis } & \multirow[b]{2}{*}{$\alpha C$} \\
\hline & & & Mín. & Máx. & 10 & $\begin{array}{c}\mathbf{2 0} \\
\text { Mediana }\end{array}$ & 3o & \\
\hline QV geral & 72,1 & 12,7 & 25,7 & 100 & 62 & 75 & 84,6 & 0,722 \\
\hline \multicolumn{9}{|l|}{ Domínios } \\
\hline Físico $¥$ & 63,7 & 23,8 & 7,1 & 100,0 & 51,8 & 67,9 & 82,1 & 0,878 \\
\hline Psicológico¥ & 66,5 & 17,6 & 29,2 & 95,8 & 56,3 & 70,8 & 77,1 & 0,805 \\
\hline Relações sociais & 72,7 & 10,6 & 50,0 & 91,7 & 66,7 & 75,0 & 79,2 & 0,636 \\
\hline Meio ambiente & 65,2 & 14,1 & 28,1 & 92,9 & 57,8 & 65,6 & 76,6 & 0,764 \\
\hline
\end{tabular}

Alpha de Cronbach $(\alpha C)$; $¥$ : variável com distribuição assimétrica (Kolmogorov Smirnov; $p<0,05$ )

Fonte: Dados dos autores.

Tabela 3 - Medidas de tendência central e de variabilidade para a escala HAD na perspectiva de estomizados. RS, Brasil. 2018

\begin{tabular}{|c|c|c|c|c|c|c|c|c|}
\hline \multirow{2}{*}{ Escalas $¥$} & \multirow{2}{*}{ Média } & \multirow{2}{*}{$\begin{array}{l}\text { Desvio } \\
\text { padrão }\end{array}$} & \multicolumn{2}{|c|}{ Amplitude } & \multicolumn{3}{|c|}{ Quartis } & \multirow{2}{*}{$\alpha C$} \\
\hline & & & Mínimo & Máximo & 10 & 2o Mediana & 3o & \\
\hline TOTAL_Ansiedade & 5,5 & 4,5 & 1,0 & 17,0 & 2,0 & 4,0 & 8,5 & 0,845 \\
\hline TOTAL_Depressão & 4,9 & 3,9 & 0,0 & 15,0 & 2,0 & 3,0 & 7,5 & 0,764 \\
\hline
\end{tabular}

¥: variável com distribuição assimétrica (Kolmogorov Smirnov; $p<0,05$ )

$$
\text { Fonte: Dados dos autores. }
$$

Quando as escalas EAGP, WHOQOL-bref, HAD tiveram suas pontuações comparadas às variáveis do perfil - sexo, estado civil, pessoas que vivem na casa, renda e tempo ostomia - não ocorreram diferenças estatisticamente significativas. Desta forma, não há evidências de relações de dependência do perfil da amostra em relação às escalas utilizadas neste estudo

Houve correlação significativa, positiva, entre EAGP e WHOQOL-bref, em que elevadas pontuações no EAGP mostraram-se correlacionadas a elevadas pontuações nos domínios Físico $\left(r_{s}=0,831 ; p<0,001\right)$ e Psicológico $\left(r_{s}=0,546 ; \mathrm{p}=0,005\right)$, bem como com a $\mathrm{QV}$ Geral $\left(r_{s}=0,716 ; p<0,001\right)$.

Com a escala de ansiedade foram detectadas correlações significativas, negativas, classificadas, em sua maioria, como moderadas, com os domínios Psicológico $\left(r_{s}=-0,562 ; p=0,004\right)$ e das Relações Sociais $\left(r_{s}=-0,575 ; \mathrm{p}=0,003\right)$, bem como com a QV Geral, $\left(r_{s}\right.$ $=-0,347 ; p=0,009)$. Dessa forma, há evidências de que elevadas pontuações na escala de ansiedade mostraram-se relacionadas a baixas pontuações nos domínios da WHOQOL-bref mencionados, ou seja, elevados escores de ansiedade relacionados a um maior comprometimento na QV.
No que se refere à escala de depressão, ocorreram correlações significativas, negativas, com grau moderado $(0,400<r<0,699)$, com todos os domínios da WHOQOL-bref, apontando que elevadas pontuações na escala da depressão mostraram-se correlacionadas a baixos escores para a QV.

$\mathrm{Na}$ comparação que envolveu a escala EAGP e a escala HAD, os resultados não foram significativos, indicando que existe uma relação de independência entre as variáveis comparadas.

Tabela 4 - Análise de correlação de Spearman entre as escalas EAGP, WHOQOL-bref e HAD. RS, Brasil. 2018

\begin{tabular}{|c|c|c|c|c|c|c|}
\hline \multirow{2}{*}{ Escalas } & \multicolumn{2}{|c|}{ EAGPT Total } & \multicolumn{2}{|c|}{ Ansiedade } & \multicolumn{2}{|c|}{ Depressão } \\
\hline & $\mathbf{r}_{s}$ & $\mathbf{P}$ & $\mathbf{r}_{s}$ & $\mathbf{P}$ & $\mathbf{r}_{s}$ & $\mathbf{P}$ \\
\hline Físico & 0,831 & $<0,001$ & $-0,308$ & 0,134 & $-0,437$ & 0,029 \\
\hline Psicológico & 0,546 & 0,005 & $-0,562$ & 0,004 & $-0,623$ & 0,001 \\
\hline Relações sociais & 0,313 & 0,128 & $-0,575$ & 0,003 & $-0,503$ & 0,010 \\
\hline Meio ambiente & 0,319 & 0,120 & $-0,090$ & 0,668 & $-0,411$ & 0,041 \\
\hline QV Geral & 0,716 & $<0,001$ & $-0,437$ & 0,009 & $-0,521$ & 0,009 \\
\hline EAGPT Total & & & $-0,189$ & 0,367 & $-0,376$ & 0,064 \\
\hline
\end{tabular}

$r_{s}$ : Coeficiente de correlação de Spearman

Fonte: Dados dos autores. 


\section{DISCUSSÃO}

É importante discutir a caracterização sociodemográfica dos participantes, tendo em vista que estes dados podem facilitar o planejamento de ações pela equipe de saúde. Esses achados corroboram a literatura, pois a incidência de neoplasias em pessoas com idade acima de 50 anos é maior, e é fator associado à ocorrência de CCR (CUNHA; FERREIRA; BACKES, 2013). Estudo realizado com estomizados identificou média de idade de 62,9 anos, com nível de escolaridade de até oito anos de estudo (70,9\%) (AGUIAR, et al., 2017). Ainda, quanto à renda familiar média, foi de 2,08 salários mínimos e, também, quanto ao tempo de estomia, a média foi de 5,3 anos (AGUIAR, et al., 2017).

As pessoas que têm estomia precisam aprender a conviver com as limitações no seu cotidiano. Nesse sentido, avaliar a autoeficácia é importante, pois ela está relacionada a resultados positivos em saúde. Pode ser compreendida em domínios específicos e em termos da confiança global diante de exigências e situações novas (QUEIROZ et al., 2017). Para os mesmos autores a autoeficácia atua como um mecanismo regulador da ação humana, influencia a capacidade de estabelecer metas, executar planos de ação, tomar decisões e autoavaliar o comportamento; também influencia o grau de esforço e persistência na busca de um objetivo e nas expectativas de resultado (QUEIROZ et al., 2017).

Indivíduos com autoeficácia elevada tendem a estabelecer objetivos mais complexos e desafiadores, explorar mais o seu ambiente e persistir na situação ou tarefa diante de obstáculos (SBICIGO et al., 2012). A autoeficácia geral pode ser um indicador útil na área da saúde, tendo em vista que tem sido um preditor de $\mathrm{QV}$, ajustamento psicossocial em portadores de doenças crônicas (SBICIGO et al., 2012).

Ainda, resultados do estudo em tela no que se refere à autoeficácia foram satisfatórios, denotando relação com a QV, com o ajustamento psicossocial, também evidenciado com os escores de ansiedade e depressão. Baixa autoeficácia está relacionada e associada com ansiedade, depressão, solidão e baixa autoestima (COELHO et al., 2016).

A QV, entretanto, tem sido um conceito multidimensional, com indicativos objetivos e subjetivos de bem-estar físico e pessoal. A avaliação objetiva está focada nos indicadores de saúde físicos e suas limitações, e a subjetiva refere-se à avaliação do indivíduo nas dimensões física, psicológica, social e ambiental. Logo, a condição de vida e saúde está atrelada a fatores como a má distribuição de renda, o analfabetismo e baixo grau de escolaridade, assim como as condições precárias de habitação e ambiente têm um papel muito importante nas condições de vida e saúde (CASTRO; PINTO; PONCIANO, 2009).

Pesquisa realizada em um centro ambulatorial da capital do Piauí, que avaliou a QV de pessoas com estomias intestinais de eliminação e verificou sua associação com características sociodemográficas e clínicas, mostrou que elas interferem na QV, principalmente nos âmbitos físico e social (ALMEIDA-BRASIL et al., 2017).

Isso demonstra que os fatores clínicos dos estomizados interferem mais na sua QV do que os fatores sociodemográficos. Fatores como condições de saúde, doenças associadas e sintomas clínicos como dor, desconforto, que limitam as atividades diárias, interferem na QV. Nesse sentido, é possível que as equipes de saúde possam intervir no que se refere aos fatores clínicos que os prejudicam, com consultas regulares, acompanhamento domiciliar, uso de correto de medicações, entre outros.

Este estudo constatou que o número de pessoas por residência variou de um a mais que três e $68 \%$ viviam com mais de três pessoas no domicílio. Outro estudo demonstrou que morar com mais de três pessoas no mesmo domicílio esteve associado a uma pior percepção da QV no domínio relações sociais (CASTRO; PINTO; PONCIANO, 2009). O Programa das Nações Unidas para os Assentamentos Humanos considera a "área suficiente para viver" uma dimensão a ser avaliada para monitoramento dos assentamentos precários, sendo preconizado não mais que duas pessoas compartilhando o mesmo dormitório, a fim de se criar um espaço adequado para o desenvolvimento das relações humanas e da interação social (CASTRO; PINTO; PONCIANO, 2009).

O domínio físico, quando comparado aos demais, apresentou a maior associação negativa entre a autopercepção de saúde como razoável ou ruim e a QV. Tal resultado pode estar relacionado à presença de doenças crônicas ou aos hábitos de estilo de vida, como o tabagismo e sedentarismo, que estão associados à autopercepção de saúde do indivíduo como ruim. A saúde pode ser determinada e condicionada, na percepção do indivíduo, por uma série de fatores determinantes da saúde, como as condições de vida e trabalho, fatores psicossociais, econômicos, culturais e comportamentos individuais (CASTRO; PINTO; PONCIANO, 2009).

O domínio físico está relacionado com as situações de dor, desconforto, energia, fadiga, sono, repouso, atividades da vida cotidiana, dependência de me- 
dicação ou de tratamentos, mobilidade, capacidade de trabalho. Assim sendo, muitos pacientes com CCR e uma estomia podem vir a ter alguma destas alterações em razão da doença e da estomia.

Nesse sentido, estudo de revisão sistemática identificou que o isolamento social, privação de sono, preocupações financeiras, inibição sexual e outras questões desse tipo são comuns entre os pacientes. Ressalta ainda as principais questões psicossociais que os pacientes com câncer enfrentam após uma estomia e sugere que os prestadores de serviços de saúde devam trabalhar estas questões para permanecer sensíveis (SILVA et al., 2017).

Pesquisa que examinou a QV relacionada à saúde em pessoas com estomias em tratamento ambulatorial e analisou a relação entre a QV, fatores clínicos demográficos e pertinentes, concluiu que os pacientes com estomia apresentaram escores totais de 69,6 \pm 20,2 para o WHOQOL-Bref. Pacientes com menos tempo de estomia obtiveram escores piores em QV. Nos pacientes que não praticavam religião e naqueles sem um parceiro aumentou a probabilidade de ter escores piores no WHOQOL-Bref (KENDERIAN; STEPHENS; JATOI, 2014).

Estudo que avaliou a QV em pacientes estomizados mostrou que o apoio do cônjuge, família e amigos influenciou positivamente. Quanto à vida sexual, $60 \%$ relataram satisfação, no entanto esse estudo não observou associação direta entre satisfação com a vida sexual e QV. Também na autoavaliação da QV, demonstraram uma melhora nesse aspecto de acordo com o tempo. A aceitação da aparência física atual foi observada em $100 \%$ dos participantes e o desempenho das atividades da vida diária não foi restringido pela bolsa coletora em nenhum dos participantes do estudo; já quanto à frequência de sentimentos negativos, $60 \%$ relataram a ocorrência de tais sentimentos em algumas situações (SANTOS; AUGUSTO; GOMBOSKI, 2016).

Nesse sentido, ao avaliar a QV em geral e seus domínios, é necessário investigar também a ansiedade e depressão de estomizados, pois esses indivíduos têm muitas alterações de imagem corporal e psicológicas. Conforme aumentam os níveis de sintomas depressivos, pior se revela a QV, com o domínio relações sociais sendo um dos três que mais se mostraram afetados.

Ter o diagnóstico de CCR e possuir uma estomia traz muitas dúvidas e inseguranças para pacientes e familiares, sendo, muitas vezes, acompanhados por transtornos psiquiátricos, que, em sua maioria, são caracterizados em dois grupos básicos: ansiedade e depressão os quais possuem alta prevalência na população brasileira (SALLES; BECKER; FARIA, 2014).

Nesse estudo os autores referem que a depressão e a ansiedade ocorrem de forma independente em 30\%-39\% dos pacientes com CCR, portanto devem ser foco de mais pesquisas, pois o diagnóstico e tratamento são muitas vezes omitidos nesses pacientes, refletindo na QV, com prejuízo na capacidade funcional e presença de limitações (SANTOS et al., 2014).

Estudo que avaliou a QV, dor, depressão e ansiedade em pacientes com câncer de reto, submetidos à intervenção cirúrgica com intenção curativa, mostrou alta prevalência de depressão ( $46,3 \%$ ) e foram evidentes as correlações entre ansiedade e depressão em virtude de problemas sexuais (SANTOS et al., 2014). Logo, há forte associação de problemas psicológicos e disfunção sexual com altos níveis de depressão, ansiedade e outras queixas negativas, associadas a manifestações somáticas. Também foi destacada a forte correlação entre depressão e perspectiva futura, havendo influência negativa da depressão na manutenção das relações interpessoais, que induz o paciente à solidão e desesperança. Também houve alta prevalência da ansiedade $(39,1 \%)$ (SANTOS et al., 2014).

Os resultados obtidos de correlações realizadas entre autoeficácia e QV em pacientes com doenças crônicas mostraram relações importantes entre essas duas variáveis, indicando que aqueles indivíduos que possuem alta autoeficácia são também os que apresentam mais alta QV em geral e na dimensão ambiente (COELHO et al., 2016).

Esses dados poderão subsidiar as equipes da rede de atenção à saúde a realizar planejamento e instituição de ações que favoreçam o cuidado, no sentido de facilitar o processo adaptativo, mediante atividades educativas direcionadas, promocionais e de diagnóstico precoce, propiciando um atendimento de qualidade e eficaz, bem como a melhoria da qualidade de vida do paciente, apoiando-se em conhecimentos científicos (QUEIROZ et al., 2017).

\section{CONSIDERAÇÕES FINAIS}

Estudo evidenciou que os estomizados apresentaram boa autoeficácia. Em relação à QV, média mais elevada ocorreu no domínio das relações sociais e médias intermediárias nos domínios psicológico, físico e meio ambiente. Pontuações elevadas de ansiedade e depressão se relacionam a um maior comprometimento na QV. Quando comparadas as escalas de autoeficácia, QV, ansiedade e depressão com as 
características sociodemográficas e clínicas, constata-se que não ocorreram diferenças estatisticamente significativas.

Houve correlação significativa, positiva, entre autoeficácia e $Q V$, em que elevadas pontuações na escala de autoeficácia mostraram-se correlacionadas a altas pontuações nos domínios físico e psicológico, bem como com a QV geral.

Com a escala de ansiedade foram detectadas correlações significativas, negativas, classificadas, em sua maioria, como moderadas, com os domínios psicológico e das relações sociais, bem como com a QV geral. Dessa forma, há evidências de que elevadas pontuações na escala de ansiedade mostraram-se relacionadas a baixas pontuações nos domínios da QV mencionados, ou seja, elevados escores de ansiedade revelam maior comprometimento na QV.

No que se refere à escala de depressão, ocorreram correlações significativas, negativas, com grau moderado, com todos os domínios da QV, observando-se que elevadas pontuações na escala da depressão mostraram-se correlacionadas a baixos escores para a QV.

Define-se como limitações deste estudo o tamanho da amostra. Novas pesquisas devem ser realizadas com esta população para constatarmos se em uma amostra maior teríamos outros resultados.

\section{REFERÊNCIAS}

AGUIAR, Janderson Cleiton et al. Aspectos sociodemográficos e clínicos de estomizados intestinais provisórios. Reme - Rev. Min. Enferm., 21:e-1013, 2017. Disponível em: http:// www.reme.org.br/artigo/detalhes/1149.

ALMEIDA-BRASIL, Celline Cardoso et al. Qualidade de vida e características associadas: aplicação do WHOQOL-BREF no contexto da Atenção Primária à Saúde. Ciência \& Saúde Coletiva, 22(5), p. 1.705-1.716, 2017. Disponível em: http:// www.scielo.br/pdf/csc/v22n5/1413-8123-csc-22-05-1705. pdf.

ATTOLINI, Raquel Cozer; GALLON, Carin Weirich. Qualidade de vida e perfil nutricional de pacientes com câncer colorretal colostomizados. Rev. Bras. Coloproct., 30 (3), p. 289-298, 2010. Disponível em: http://www.scielo.br/pdf/rbc/v30n3/ a04v30n3.pdf.

BOTEGA, Neury J. et al. Transtornos do humor em enfermaria de clínica médica e validação de escala de medida (HAD) de ansiedade e depressão. Rev. Saúde Pública, 29 (5), p. 355-63, 1995. Disponível em: http://www.scielo.br/pdf/ rsp/v29n5/04.pdf.

BRASIL. Ministério da Saúde. Instituto Nacional de Câncer. Estimativa 2018: incidência de câncer no Brasil. 2018. Disponível em: https://www.inca.gov.br/publicacoes/livros/ estimativa-2018-incidencia-de-cancer-no-brasil.
CASTRO, Elisa Kern de; PINTO, Débora Wagner; PONCIANO, Clarissa Franco. Auto-eficácia e qualidade de vida de jovens adultos com doenças crônicas. Rev. SBPH, 12 (2), 2009. Disponível em: http://pepsic.bvsalud.org/pdf/rsbph/v12n2/ v12n2a09.pdf.

COELHO, Eduardo et al. Autoeficácia e qualidade de vida no trabalho: um estudo com policiais militares. Psic.: Teor. e Pesq., 32, p. 1-7, 2016. Disponível em: http://www.scielo. $\mathrm{br} / \mathrm{pdf} / \mathrm{ptp} / \mathrm{v32nspe/1806-3446-ptp-32-spe-e32ne220.pdf.}$

CUNHA, Regina Ribeiro; FERREIRA, Artur Brandão; BACKES, Vânia Marli Schubert. Características sócio-demográficas e clínicas de pessoas estomizadas. Revista Estima, 11 (2), p. 327, 2013. Disponível em: https://www.revistaestima.com. br/index.php/estima/article/view/327.

FLECK, Marcelo P. A. et al. Aplicação da versão em português do instrumento abreviado de avaliação da qualidade de vida "WHOQOL-bref". Rev. Saúde Pública, 34(2), p. 17883, 2000. Disponível em: http://www.scielo.br/pdf/rsp/ v34n2/1954.pdf.

GOMBOSKI, Gustavo. Adaptação cultural e validação do City of Hope - Quality of Life - Ostomy Questionnaire para a língua portuguesa no Brasil. 2010. Dissertação (Mestrado em Enfermagem) -Escola de Enfermagem da Universidade de São Paulo, 2010. Disponível em: http://www.teses.usp. br/teses/disponiveis/7/7139/tde-01082011-074203/pt-br. php.

KENDERIAN, S.; STEPHENS, E. K.; JATOI, A. Ostomies in rectal cancer patients: what is their psychosocial impact? European Journal of Cancer Care, 23, p. 328-332, 2014. Disponível em: https://onlinelibrary.wiley.com/doi/epdf/10.1111/ ecc.12133.

LUZ, Alyne Leal de Alencar et al. Perfil de pacientes estomizados: revisão integrativa da literatura. Cultura de los Cuidados, 18 (39), p. 115-123, 2014. Disponível em: https:// rua.ua.es/dspace/bitstream/10045/40073/1/Cultura_Cuidados_39_13.pdf.

MARCOLINO, José Álvaro Marques et al. Escala hospitalar de ansiedade e depressão: estudo da validade de critério e da confiabilidade com pacientes no pré-operatório. Revista Brasileira de Anestesiologia, 57: 1, p. 52-62, 2007. Disponível em: http://www.scielo.br/pdf/\%0D/rba/v57n1/06.pdf.

MIRANDA, Sara Machado et al. Caracterização sociodemográfica e clínica de pessoas com estomia em Teresina. Revista Estima, 14 (1), p. 29-35, 2016. Disponível em: https://www.revistaestima.com.br/index.php/estima/article/ view/117/0.

MONTEIRO, Sandra de Nazaré Costa et al. Caracterização de cuidadores de crianças e adolescentes estomizados atendidos em serviço de reabilitação. Revista Estima, 14 (2), p. 7683, 2016. Disponível em: https://www.revistaestima.com. br/index.php/estima/article/view/380.

PATRÃO, Ana Luisa; ALVES, Vicente Paulo; NEIVA, Tiago. Propriedades psicométricas da escala de auto eficácia geral em idosos brasileiros. Psicologia Saúde \& Doenças, 18(1), p. 29-38, 2017. Disponível em: http://www.scielo.mec.pt/pdf/ psd/v18n1/v18n1a03.pdf. 
PLETSCH, Graziela Meneghelli Cabrelli. Avaliação da qualidade de vida de pacientes estomizados no município de Teutônia. 2013. Trabalho (Conclusão de Curso de Especialização em Estomaterapia) - Universidade do Vale do Rio dos Sinos, Unisinos, 2013. Disponível em: http://www.repositorio.jesuita.org.br/bitstream/handle/UNISINOS/5655/Graziela\%20Meneghelli\%20Cabrelli\%20Pletsch_.pdf?sequence=1\&isAllowed $=y$.

QUEIROZ, Cíntia Galvão et al. Caracterización de ileostomizados atendidos en un servicio de referência de ostomizados. Enfermería Global, 46, 2017. Disponível em: http://scielo.isciii.es/pdf/eg/v16n46/pt_1695-6141eg-16-46-00001.pdf.

RIBEIRO, Raíssa Vanessa Lima et al. Adaptação social do paciente colostomizado: desafios na assistência de enfermagem. R. Interd., 9 (2), p. 216-222, 2016. Disponível em: https://revistainterdisciplinar.uninovafapi.edu.br/index.php/ revinter/article/view/1128/pdf_329.

SALLES, Valdemir José Alegre; BECKER, Carolina de Paula Penteado; FARIA, Giuliana da Matta Rossi. The influence of time on the quality of life of patients with intestinal stoma. J Coloproctol., 34(2), p. 73-75, 2014. Disponível em: http://www.scielo.br/pdf/jcol/v34n2/2237-9363-jcol-34-02-00073.pdf.

SANTOS, Letácio José Freire et al. Quality of life, pain, anxiety and depression in patients surgically treated with cancer of rectum. ABCD Arq. Bras. Cir. Dig., 27(2), p. 96-100, 2014. Disponível em: http://www.scielo.br/pdf/abcd/v27n2/ 0102-6720-abcd-27-02-00096.pdf.

SANTOS, Vera Lucia Conceição de Gouveia, AUGUSTO, Fabiana da Silva; GOMBOSKI, Gustavo. Health-Related Quality of Life in Persons With Ostomies Managed in an Outpatient Care Setting. J Wound Ostomy Continence Nurs, 43(2), p. 158-164, 2016. Disponível em: https://www.ncbi.nlm.nih. gov/pubmed/26808303.

SBICIGO, Juliana Burges et al. Propriedades psicométricas da escala de autoeficácia geral percebida. Psico., 43 (2), p. 139146, 2012. Disponível em: http://revistaseletronicas.pucrs. br/ojs/index.php/revistapsico/article/view/11691/8037.

SILVA, Cynthia Roberta Dias Torres et al. Qualidade de vida de pessoas com estomias intestinais de eliminação. Acta Paul Enferm., 30(2), p. 144-51, 2017. Disponível em: http:// www.scielo.br/pdf/ape/v30n2/1982-0194-ape-30-02-0144. pdf.

SOUZA, Israel; SOUZA, Marcos Aguiar de. Validação da escala de auto eficácia geral percebida. Rev. Univ. Rural, Sér. Ciências Humanas, 26 (1-2), p. 12-17, 2004. Disponível em: https://www.researchgate.net/publication/260338439_Validacao_da_Escala_de_Autoeficacia_Geral_Percebida. 